\title{
Survey on Quick Response Code
}

\author{
Revanth kumar U \\ UG Student, \\ Department of CSE, \\ S.A Engineering College \\ Chennai - 600077
}

\author{
Prasanth J \\ UG Student, \\ Department of CSE, \\ S.A Engineering College \\ Chennai - 600077
}

\author{
Prashanth R \\ UG Student, \\ Department of CSE, \\ S.A Engineering College \\ Chennai - 600077
}

\author{
Ms. Elavarasi K \\ Assistant Professor, \\ Department of CSE, \\ S.A Engineering College \\ Chennai - 600077
}

\author{
Ms. Jayanthi K \\ Assistant Professor, \\ Department of CSE, \\ S.A Engineering College \\ Chennai - 600077
}

\begin{abstract}
QR code is abbreviated as Quick Response Code and it is truncated as snappy reaction code, generally $\mathrm{QR}$ code is utilized just in outside nations .QR code can be checked in 360 degree headings. QR codes are utilized as a part of the enormous enterprises on account of its bigger stockpiling limit and high harm resistance than the standardized tags. It can be checked in both flat and vertical headings. A straightforward output catches the fancied data. The Decoded information can be put away in the server and can be seen by the clerk. Deciphering calculation is utilized to examine the QR codes in versatile. QR Code can't without much of a stretch be perused with the bare eye so they especially hard to deal with. Two QR Codes with various activities will never be the same. There are absolutely two unique examples in QR Codes. So QR Codes is impractical to be hacked.
\end{abstract}

KEYWORDS: QR Code, Decoding Algorithm, Android, Mobile Scanner, Bar code.

\section{INTRODUCTION}

Data mining is the process of analysing data from different sources and differentperceptive into a useful information. Thus the information is nothing but a usage of revenue and cost of the certain product. It is used to identify the user in a way of different dimension and appropriate angles inorder to attain various measurements based on our requirements. It is also can be stated as finding the correlation of relation between several databases.

These concepts were used on many platforms in order to make it on existing resource and to use these products on online for various customers. The major involvement of these data is based on the way in which validators and end users meet the need of concern area.

\subsection{Foundation of Data mining}

Data mining takes the responsibility in a way of storage purposes of computer and later it is onmany applications and at the same time it is for business requirements to satisfy the needs of various users. There are many algorithm were founded for this kind of data information because they are build up real world system products. The massively parallel processing is one of the key source for these foundation and different multiprocessor were also been attained towards these systems. A group of survey was reported that foundation of data warehouse bought into the existence about $23 \%$ in the Mideast countries. In the business needs they are kind of buildingblock for various projects to get completed and there each and every step made the developer to invent newer software which can be used at all source of the products.

\subsection{Data}

Data is nothing but a collection of information, facts and many text formats in order to collect and process over the computer. They are used at the several organization to storing purposes, now a days the big data plays a vital role many industries which comprises many things in it, for example meta data, operational data etc.

\subsection{Information}

The collection, patterns and coordination in all these data gives the information. For example if you're buying a product you will gather certain information regarding that products price manufacturing date and etc.

\subsection{Data Warehouse}

It is defined as the process of centralized data and processing it to a different ways and retrieving it. Advancing the user to use the data at quicker time without any problems towards their requirements.

\subsection{Data Mining Working}

Data mining analyzes the relationship between various data and gathers proper information with the transaction database to the open end user satisfaction. There are different software available for data to get process they are, statistical, machine learning method, neural networks. 


\subsection{Classes}

The data which is used in predetermined groups for accessing. For example, in a motel there will be several customers and any one can order any dishes, thus this kind of information may increase the motel traffic methodology.

\section{OLTP (online transactional processing)}

This technology used for performing updates on operational and transactional systems. They are mainly used for customer management and satisfying the user queries.

\section{OLAP (online analytical processing)}

It is used to perform complex analysis of data in data warehouse OLAP queries are executed over a separate copy of the working data in which the process overlap.

\section{Types:}

1. ROLAP (Relational OLAP),

2. MOLAP (Multidimensional OLAP),

3. HOLAP (ROLAP + MOLAP).

\section{LITERATURE SURVEY:}

\subsection{ISO/IEC 15420:2009. Information} technology - Automatic identification and data capture techniques - EAN/UPC bar code symbology specification. 2009.

QR Code is a lattice symbology comprising of a variety of ostensibly square modules masterminded in a general square example, including an interesting discoverer design situated at three corners of the image and expected to help with simple area of its position, size and slant. An extensive variety of sizes of image is furnished for together with four levels of blunder redress. Module measurements are client indicated to empower image generation by a wide assortment of methods. QR Code Model 1 is the first detail for QR Code; QR Code Model 2 is an improved type of the symbology with extra components and can be autosegregated from Model 1. Since Model 2 is the prescribed model for new, open frameworks use of QR Code, this International Standard depicts Model 2 completely, and indicates the components in which Model 1 QR Code varies from Model 2 in an attach.

\subsection{Z. Baharav and R. Kakarala. Visually} significant $Q R$ codes: Image blending and statistical analysis. In Multimedia and Expo (ICME), 2013 IEEE International Conference on, pages 1-6. IEEE, 2013.

Normally the characters, numbers and so on are implanting in $\mathrm{QR}$ codes. This paper presents the idea of shading picture embedding's in QR codes. This is a programmed technique to insert QR codes into shading pictures with limited likelihood of discovery mistake. These embedding's perfect with standard interpreting applications and can be connected to any shading picture with full territory scope. To relieve the visual contortion of the QR picture, the calculation uses half toning covers for the determination of altered pixels and nonlinear programming methods to locally streamline luminance levels Take one shading picture and changed over into dark picture. At that point this doing the concealing procedure, window extraction, picture installing, interpreting like procedures. After this procedure the first dark picture is taken from this.

\subsection{Baras and F. Cayre. 2D bar-codes for authentication: A security approach. In Signal Processing Conference (EUSIPCO), Proceedings of the 20th European, pages 1760-1766, 2012.}

In this paper, we check the confirmation issue of genuine products on which 2D standardized identifications in the perspective of adversary. Expect that the adversary has the entrance to Noise Criteria boisterous duplicates of particular $2 \mathrm{D}$ standardized tags. The commotion is because of printing and filtering forms. An estimator of 2D scanner tags are based upon the duplicates that is proposed, permitting the adversary to make a copy 2D standardized identification which goes for being announced as particular by the framework finder. The execution of the estimator is inferred as for Noise Criteria regarding mistake likelihood at the locator side and contrasted and the useful outcomes on unique 2D standardized tags. It is demonstrated that the rival can make a copy that effectively imagines the finder with a sensible number of products.

\subsection{QR Codes and the Dialogue Between Analogue and Digital}

The QR Code is an imaginative expansion of the standard scanner tag found on each fabricated item, for example, sustenance's, books and tickets. The essential preferred standpoint about the QR Code is that they can convey more data in a littler space, since they can be perused in both vertical and even positions. They can be perused at different edges, with the calculation used to make them empowering a higher room for give and take in the vicinity of 7 and 30 $\%$. The capacity to filter QR Codes requires of a cell phone with an Internet association, a camera and a telephone application that will perceive and interpret the QR Codes. All the "cell phones" that are fabricated have this capacity. Advanced mobile phone clients can experience QR Codes for all intents and purposes anyplace. The most widely recognized areas incorporate at inside stores, home and different organizations, on pathways and walkways through notices, store windows, and keeping in mind that going on open transportation and at work. The QR Code can store the data and impressions about the Products.

\subsection{Decoding Algorithm for color QR code: A Mobile Scanner Application}

The QR Code has reformed the enterprises because of its focal points, for example, bigger capacity limit and more noteworthy harm resistance than the ordinarily utilized standardized identifications. Shading QR code is a framework that it gives the bigger encoding limit that the typical QR codes. The shading QR code interpreting calculation for portable application is by considering the example hues Red, Green and Blue. Contrasting with the typical standardized identifications, the QR codes can have 10 times more information in a similar measure of space. The QR code limit can be enhanced by encoding different distinctive layers of codes by different hues. The information put away in the hued QR code is specifically corresponding to $\log 2 \mathrm{~N}$. Where the $\mathrm{N}$ is the quantity of hues utilized as a part of the example. More quantities of hues can be utilized 
as a part of shading QR code. The QR code has special qualities that are capacity of blunderrectification which guarantees compelling unraveling done notwithstanding when the QR code picture is harmed, obscure or filthy. The other preferred standpoint of $\mathrm{QR}$ code is that it can be perused from any bearing. The created QR code is inPNG design. The distinctive hued QR code can be combined for further era of QR code with the assistance of arithmetical summons. In this paper we have made a cell phone application for shading QR codes.

2.6 Bingsheng Zhanginthe proposed arrangement of Short range correspondence advances including close field correspondence and with 2D scanner tags in cell phone applications. Advanced in the Radio recurrence distinguishing proof (RFID). 2D standardized tags have essentially high entrance rate in versatile correspondence. $2 \mathrm{D}$ standardized tags are much utilized for security-touchy applications including installments and individual distinguishing proof. The scanner tag based correspondence utilizes optical machine-clear example for exchanging data. There are 40 unique forms in standard QR code extending from $21 * 21$ to $177 * 177$ modules and four blunder adjusting level (ECl) and furthermore work in mistake amendment code separately. The Visible Light Communication has duplex channel. There are 3 secure correspondence conspire.

- $\quad$ Two stage message exchange conspire

- Smartphone handshake conspire

- All-or-nothing information gushing plan

The sender and the collector of the SBVLC correspondence mode may not anticipated that would have a same sharing mystery key. The information trade in SBVLC underpins for both cell phone to cell phone and cell phone to terminal circumstances. SBVLC works with full duplex VLC channel and in this way the cell phone must have shading screen and auxiliary camera with high determination for correspondence amongst sender and collector.

2.7 Dijana Jagodiin the proposed arrangement of android based framework for perusing Quick Response (QR) Code. QR code is a two dimensional image yet Barcode is a one dimensional image. Information put away in the $Q R$ code is not effectively getting it. It gives additional layers for more security. QR code is so mainstream all in all open used to interface between physical world and advanced world. QR codes gives more security it needs just camera in the portable or tablet to check the code. The structure of the QR code as square lattice. The structure can be in distinguished in 360 degree bearings. It is anything but difficult to distinguish the code. The QR code information are encoded in the Paired frame ' 0 's and ' 1 's. Those numbers are changed over into high contrast cells and masterminded later.

\subsection{Siti Fatimah Abdul Razak, Choon Lin} Liew, Chin Poo Lee, Kian Ming Lim, the proposed arrangement of android based indoor stopping framework with the assistance of QR code tells about the android application for stopping the vehicle so as to diminish the movement clog and furthermore for client fulfillment. Here we will utilize the java code and SQL database design for certain association. For instance if you're setting off to a shopping center by your vehicle and in a few conditions there will be numerous issues like more number of guests could may come so keeping in mind the end goal to stay away from that sort of issue we have presented this android application. Here we have guide of our area and the route alternative additionally will be accessible by utilizing it we can see the accessible spot for our vehicle, there is no need of username and secret key for each and person. The movement sensor and camera which will be accessible in our android gadgets and assumes a noteworthy part in our venture.

\subsection{Peter KiesebergManuel Leithner,} Martin Mulazzani, the proposed arrangement of QR code for security is utilized for the assurance of different use and it is additionally utilized by many individuals and it is concentrating on the people groups keeping in mind the end goal to safe our valuable needs which we are utilizing as a part of our day by day life. The mostly utilized one in this venture is SQL summons in light of the fact that QR code is a one of a kind one the blunder remedy will likewise be done as such we can without much of a stretch recognize the issue emerging. Here the shading will be distinctive for every last information and it will demonstrate the malware which is been robbery. They can be of any sort however the fundamental reason on the planet is weakness so this venture of QR code will be utilized as a part of all kind of utilization in our day by day life.

\section{CONCLUSION}

Hence this review paper tells about different utilization of QR code and it counter measures in different environment, in this paper different creator told about their own particular venture in regards to QR code security and its burdens and assaults it has and so on at last we propose an arrangement of security countermeasures that can be received in orderto handle the security issues tended to in our exploration. Computerized strategies alongside recommendations for legitimate client training are incorporated into our arrangement of countermeasures. These countermeasures have not been analyzed inside and out yet, but rather they can frame a strong base where additionally research can be sent.

\section{REFERENCES}

[1]Peter Kieseberg, Manuel Leithner, Martin Mulazzani, Lindsay Munroe, SBA Research Favoritenstrasse 16 AT1040 Vienna, Austria

[2] Information technology - Automatic identification and data capture techniques - EAN/UPC bar code symbology specification. 2009.

[3] Information technology - Automatic identification and data capture specification. 2006.

[4]Z. Baharav and R. Kakarala. Visually significant QR codes: Image blending and statistical analysis. In Multimedia and Expo (ICME), 2013 IEEE International Conference

[5]C. Baras and F. Cayre. 2D bar-codes for authentication: A security approach. In Signal Processing Conference (EUSIPCO)

[6] "Evaluating Voting Technology," Douglas W. Jones, Testimony before the United States Civil Rights Commission, Tallahassee, Florida, January 11, 2001 
[7] Decoding Algorithm for color QR code:A Mobile Scanner Application Nivedan Bhardwaj, Ritesh Kumar, Rupali Verma, Alka Jindal and Amol P. Bhondekar.

[8] Secure Barcode-based Visible Light Communication for Smartphones Bingsheng Zhang, Kui Ren, Senior Member, IEEE, Guoliang Xing, Senior Member, IEEE, Xinwen Fu, Senior Member, IEEE, and Cong Wang, Member, IEEE

[9] Interactive Android-Based Indoor Parking Lot Vehicle Locator Using QR-code Siti Fatimah Abdul Razak, Choon

Lin Liew, Faculty of Information Science and Technology Multimedia University Jln Ayer Keroh Lama, 75450 Bukit

Beruang, Melaka, Malaysia 